\section{Bias in the COVID-19 era}

\section{DOI: $10.7861 /$ clinmed.Let.21.1.4}

Editor - We thank Kelly et al for an informative and useful article. ${ }^{1}$ There is only a short mention of clinical biases, and I believe that this point needs further attention. Cognitive biases are omnipresent in medicine. They can affect reasoning and result in adverse events. ${ }^{2} \mathrm{~A}$ few types of biases exist here. One was confirmation bias which is the analysis of information in a particular way to confirm and strengthen original suspicions or hypotheses. This was most probably in play on the patient's first presentation where the diagnosis of 'mild COVID-19' was entertained. There is also clear availability bias, this is an intrinsic human tendency where one assesses the likelihood of an event by how often other examples come to one's mind, thus creating mental shortcuts. Anchoring bias was also at play, this is where one settles on a diagnosis based on a few important features. It is difficult to know if affective bias was present, which is the tendency to convince oneself what one wants to be true. It is also easy to perform a retrospective analysis of any case such as this one and this is by no means a criticism of the initial treatment, but COVID-19 mimics are increasingly being described. ${ }^{3}$ Good clinical acumen and an understanding of cognitive biases (which are not taught at medical school and only come from continuous adult learning and reflective practice) will make sure that patients continue to receive good medical care.

AVINASH AUJAYEB

Consultant in respiratory and acute medicine, Northumbria Healthcare NHS Foundation Trust, Cramlington, UK

\section{References}

1 Kelly S, Waters L, Cevik M et al. Pneumocystis pneumonia, a COVID-19 mimic, reminds us of the importance of HIV testing in COVID-19. Clin Med 2020;20:590-2.

2 O'Sullivan ED, Schofield SJ. Cognitive bias in clinical medicine. J $R$ Coll Physicians Edinb 2018:48:225-32.

3 Hanfi SH, Lalani TK, Saghir A et al. COVID-19 and its mimics: what the radiologist needs to know. J Thorac Imaging 2020 [Epub ahead of print].

\section{General management approach to common vasculitides}

\section{DOI: $10.7861 /$ clinmed.Let.21.1.5}

Editor - We recently read the article, 'An update on the general management approach to common vasculitides' by Hng, Zhao and Moots. ${ }^{1}$

We would like to suggest refinements of the description of dermatological manifestations in a patient with probable vasculitides as outlined their Table 2 and Fig 2, as we are concerned that comments like 'new persistent rash' might be a little too generalised and non-specific.

This issue is important to be addressed since there have been significant updates of the classification of vasculitis and dermatologists have strived to define lesions of vasculitis in patients with greater accuracy, and the Chapel Hill Consensus of 2018 reflects those efforts. ${ }^{2}$

In very simple terms, skin examination findings in vasculitis relate to vessel size. Small vessel vasculitis causes a palpable petechial rash. Medium vessel involvement on the other hand leads to livedo reticularis (a lace like discoloration of skin), ulceration, nodules or macules; while large vessel involvement leads to skin necrosis and ulceration.

We hope our comments are useful and add to the educational aspects of the paper.

SHRIYA KACHRU

Clinical attachee in general internal medicine, Queen Alexandra Hospital, Cosham, UK

ALEXA SHIPMAN

Consultant dermatologist, Queen Alexandra Hospital, Cosham, UK

\section{References}

1 Hng M, Zhao SS, Moots RJ. An update on the general management approach to common vasculitides. Clin Med 2020;20:572-9.

2 Sunderkötter $\mathrm{CH}$, Zelger B, Chen KR et al. Nomenclature of Cutaneous Vasculitis: Dermatologic Addendum to the 2012 Revised International Chapel Hill Consensus Conference Nomenclature of Vasculitides. Arthritis Rheumatol 2018;70:171-84.

\section{HIV testing during the COVID-19 pandemic}

\section{DOI: 10.7861/clinmed.Let.21.1.6}

Editor - We support the recommendations made in the recent report by Kelly et al for increased awareness of importance of HIV testing during the COVID-19 pandemic. ${ }^{1}$

During the UK's first wave, our hospital trust in north west London experienced a high burden of COVID-19 patients, with clinical staff quickly becoming familiar with the clinical and radiological features of COVID-19. Studies that have compared pooled nasopharyngeal aspirate results with cross-sectional imaging indicate that the sensitivity of reverse transcriptase polymerase chain reaction (RT-PCR) for SARS-CoV-2 may be very poor. ${ }^{2}$ As a result, a significant proportion of COVID-19 patients are diagnosed on the basis of typical clinical and radiological features, despite negative tests. ${ }^{3}$

Between March and June 2020, three patients were admitted with signs and symptoms consistent with COVID-19 (respiratory failure and bilateral infiltrates on chest imaging) but were COVID-19 RT-PCR negative. These patients were subsequently diagnosed with advanced HIV (mean CD 4 count $62 \times 10^{6} / \mathrm{L}$ (range $\left.12-108 \times 10^{6} / \mathrm{L}\right)$ ) and Pneumocystis jirovecii pneumonia (PCP). Due to the initial presumed clinical diagnosis of COVID-19 there was a delay in testing for HIV and diagnosing and treating PCP.

PCP and COVID-19 are difficult to distinguish on clinical grounds, with shared symptoms including fever, dyspnoea, dry cough and fatigue; although, in COVID-19 the onset is more acute. ${ }^{4,5}$ The similar radiological findings include bilateral infiltrates with COVID-19 typically involving the lung peripheries whereas PCP is often peripherally sparing. ${ }^{5}$

Our experience highlights the importance of maintaining diagnostic vigilance when pursuing a diagnosis of COVID-19. We strongly advocate HIV testing all individuals presenting with acute respiratory illness, including those with 'typical' features of COVID-19 as per UK national guidelines. ${ }^{6}$

SARAH ANN FILSON

Infectious diseases and microbiology registrar, London North West University Healthcare NHS Trust, London, UK 